\title{
Un gène isolé chez la souris soumis à empreinte parentale siège chez l'homme dans la région du syndrome de Prader-Willi
}

Parmi les maladies qui paraissent liées à l'existence d'une empreinte parentale, c'est-à-dire exprimée par un seul des deux parents, seul le syndrome de Prader-Willi (SPW) est bien caractérisé. On sait qu'il résulte de l'absence d'une région située en $15 q$ 11-13 sur le chromosome d'origine paternelle, région soit délétée, soit remplacée par son homologue maternel (disomie maternelle) (voir $\mathrm{m} / \mathrm{s} n^{\circ} 7$, vol. 8, p. 741). On sait aussi qu'une maladie différente, le syndrome d'Angelman (SA) résulte, souvent mais non toujours, d'une anomalie symétrique, où manque la même région d'origine maternelle. Une dissection moléculaire fine de cette zone suggère que les deux régions, responsables de SPW et de SA, sont voisines mais ne coïncident pas [1]. Un progrès très important vient d'être accompli, avec la découverte, chez la souris, d'un gène correspondant à la région SPW, soumis à empreinte parentale, et de l'extension de cette découverte à l'espèce humaine. Sur les 11 effets d'empreinte parentale relatés sur l'ensemble du génome de la souris, trois sont liés au chromosome 7 ; notamment, deux gènes, $\mathrm{H} 19$ et le facteur de croissance insulin-like 2 présentent cet effet. Toutefois la région centrale de ce chromosome n'avait pas été encore explorée. On s'est donc attaché à découvrir des duplications paternelles ou maternelles de cette région centrale et à rechercher si elles étaient causes de phénomènes pathologiques. Trois articles viennent de paraître dans Nature Genetics, décrivant un gène soumis à empreinte parentale chez la souris et les conséquences chez l'homme. Leff et al. (Stanford, CA et Frederick, MD, USA) se sont intéressés à des gènes de la souris qui interviennent dans l'épissage d'ARNm spécifiques de tissus, et qui codent pour
Certaines de ces nucléoprotéines peuvent être caractérisées, car elles se comportent comme des antigènes contre lesquels se forment des anticorps chez des malades atteints de lupus érythémateux. On a ainsi pu cloner [3] un gène d'un polypeptide abondant dans le cerveau, susceptible de jouer un rôle dans l'épissage de messagers de ce tissu (snRPN, gène Snrpn).

Pour la recherche de la localisation du gène, on a utilisé, comme on le fait fréquemment en génétique de la souris, le croisement entre Mus musculus et Mus spretus, pour lesquels on connaît plus de 1000 différences. On a ainsi pu placer le gène sur la partie centrale du chromosome 7, avec un pseudogène sur le 14 . Cette région est homologue du chromosome humain 15; l'étape suivante fut de déterminer si Snrnp est soumis à empreinte parentale, en examinant les ARNm extraits de cerveaux d'hybrides $M$. musculus- $M$. spretus, qu'on peut distinguer par protection à la RNAse. Dans tous les cas, c'est seulement l'allèle paternel qui était exprimé dans le cerveau de rats nouveau-nés. Une autre méthode, employée par Cattanach et al. [4], a consisté à obtenir expérimentalement des souris présentant une duplication de la région centrale du 7 pour un segment paternel ou maternel. Une duplication maternelle provoquait des troubles de croissance aboutissant à la mort, qu'une duplication paternelle n'entraînait pas. Un gène très voisin, qui se trouve dans une région en cause dans le syndrome d'Angelman, est Gabrb3 (récepteur $\beta 3$ du GABA); il était exempt d'empreinte parentale. De l'ensemble de ces travaux ressort la conclusion que l'on peut mettre en évidence, par construction de duplications spécifiques du chromosome 7 , des anomalies rappelant le SPW mais non le SA.
Forts de ces résultats, Ozçelik et al. [5] ont entrepris de les transporter à l'espèce humaine. Par l'analyse d'hybrides cellulaires ne contenant qu'un chromosome humain, deux chromosomes se montrèrent positifs, le 6 et le 15. Le locus sur le 6 est dépourvu d'introns et correspond à un pseudogène. Sur le 15 se trouve, en revanche, un gène contenant des introns, localisé entre 15pter et q14, cette région correspond aux loci de SPW et SA. Chez sept sujets atteints de SPW avec délétions en 15q 11-13, le gène SNRPN était délété. L'étude de 2 malades porteurs d'une délétion partielle permet de définir un intervalle d'environ $1 \mathrm{Mb}$ dans lequel doit se trouver le gène SNRPN. Au contraire, dans une famille atteinte de SA, une délétion d'origine maternelle n'englobait pas le locus SNRPN. On arrive ainsi à une conclusion remarquable : la région 15q 11-13 provoque un SPW en cas de déficit génique d'origine paternelle, un SA en cas de déficit maternel ; il y a empreinte parentale dans les deux cas, et pourtant les deux régions ne coïncident pas. Le gène SNRPN est spécifique de la zone SPW ; la preuve de son caractère imprimé n'est pas encore apportée chez l'homme, il n'est qu'inféré d'après la souris. Le statut de gène candidat qui lui est ainsi conféré implique encore nombre de vérifications. Quant au gène codant pour Gabrb3, il semble spécifique de la région du SA, n'est pas englobé dans celle du $\mathrm{SPW}$, et ne semble pas soumis à empreinte. Le mécanisme par lequel une altération de SNRPN pourrait intervenir ne peut être qu'hypothétique ; la disparition de son expression dans les neurones pourrait retentir sur la maturation des messagers neuronaux. Il paraît cependant improbable que l'ensemble des manifestations 
pathologiques du SPW soit due uniquement à l'inactivation de ce seul gène. On doit donc continuer à chercher d'autres gènes impliqués dans la zone critique du chromosome 15. C'est ainsi, par exemple, que vient d'être analysé, sur le chromosome 7 de la souris, le gène $\mathrm{p}$ (pink-eyed dilution), dont le correspondant humain $\mathrm{P}$ se localise en 15 q 11-13, qui peut être muté dans l'albinisme oculocutané de type II (tyrosinase-positif), et pourrait donc être responsable de l'hypopigmentation souvent observée dans SPW et SA [6].

J.C.D.

1. Kuwano A, Mutirangura A, Dittrich B, at al. Molecular dissection of the Prader-Willi/Angelman syndrome region (15q11-13) by YAC cloning and FISH analysis. Hum Mol Genet 1992 ; 1 : 417-25. 2. I eff SE, Brannan CI, Reed ML, et al. Maternal imprinting of the mouse Snrpn gene and conserved linkage homology with the human Prader-Willi syndrome region. Nature Genet 1992 ; 2 : 259-64.

3. McAllister G, Roby-Shemkovitz A, Amara $\mathrm{SG}$, Lerner MRR cDNA sequence of the rat $U$ snRPN associated protein $\mathbf{N}$ : description of a potential $\mathrm{Sm}$ epitope. $E M B O J 1989 ; 8$ : 1177-81.

4. Cattanach BM, Barr JA, Evans EP, et al. A candidate mouse model for Prader-Willi syndrome which shows an absence of Snrpn expression. Nature Genet 1992 ; 2 : 270-4.

5. Osçelik T, I eff S, Robenson W, et al. Small nuclear ribonucleoprotein polypeptide $\mathrm{N}$ (SNRPN), an expressed gene in the Prader-Willi syndrome critical region. Nature Genet 1992 ; 2 : 265-9.

6. Rinchik EM, Bultman SJ, Horsthemke B, et al. A gene for the mouse pink-eyed dilution locus and for human type II oculocutaneous albinism. Nature 1993 ; 361 : 72-6.

\section{DD BRÈVE}

1. Administration in vivo d'oligonucléotides méthylphosphonates anticMYC à des souris transgéniques lymphomateuses. L'oncogenèse ciblée par transgénèse chez la souris a permis d'obtenir un grand nombre de modèles de cancers qui commencent à s'imposer comme des systèmes privilégiés pour élaborer et tester de nouvelles approches thérapeutiques. Parmi celles-ci, l'utilisation d'oligonucléotides antisens capables de venir inhiber spécifiquement l'expression d'un oncogène impliqué dans l'apparition d'une tumeur retient l'attention du fait de la simplicité potentielle de sa mise en œuvre et de l'inocuité qu'on lui suppose. Mais pour être actifs in vivo, encore faut-il que les oligonucléotides aient pu atteindre leur cible. Wickstrom et al., (Université de Floride du Sud, FL, États-Unis) ont évalué la stabilité d'oligonucléotides modifiés (ADN méthylphosphonates) anti-c-myc administrés par voie intraveineuse à des souris transgéniques qui développent des lymphomes B sous l'effet de l'expression du transgène $\mathrm{E} \boldsymbol{\mu}-\mathrm{c}-\mathrm{m} y \boldsymbol{c}$ [1]. Ils démontrent : (1) l'absence de toxicité des ADN méthylphosphonates anti-myc aux doses injectées $(300 \mathrm{nmol}$ soit 50 à $75 \mathrm{mg} / \mathrm{kg}$ ) ; (2) la rapide clairance des oligonucléotides à partir du sérum : (3) la diminution de la quantité d'antigène c-MYC dans les lymphocytes de souris transgéniques $\mathrm{E} \mu-c-m y \kappa$; (4) l'absence de modification des niveaux d'ARN $c$-myc. Ce dernier résultat confirme l'absence d'activité RNase $\mathrm{H}$ des oligonucléotides méthylphosphonates et suggère qu'ils agissent en bloquant la traduction des ARN auxquels ils s'hybrident et non en les dégradant. Certes, l'efficacité antilymphomateuse d'une telle approche est encore loin d'être prouvée, mais ces travaux préliminaires soulignent tout l'intérêt de la transgénèse pour obtenir le modèle le plus spécifiquement adapté au test d'une approche thérapeutique donnée.

[1. Wickstrom E, et al. Cancer Res $1992 ; 52: 6741-5$.] 